\title{
REFLEXIONES ACERCA DE LA ENSEÑANZA EN LA SOCIEDAD
}

ACTUAL

\section{REFLECTIONS ABOUT EDUCATION IN CONTEMPORARY SOCIETY}

\author{
AUTORA \\ Beatriz Peña Acuña. \\ Universidad Católica San Antonio. Murcia (España). \\ bpena@pdi.ucam.edu
}

\section{RESUMEN}

Está dentro del deseo primigenio de la UE que cualquier mejora de la educación y la formación contribuye a reforzar el crecimiento y la competitividad de Europa mientras Iberoamérica toma nota de EE.UU y Europa. En el viejo continente se destaca en repetidas ocasiones la necesidad de no limitarse a los aspectos puramente económicos del desarrollo, sino a considerar la realización personal del individuo como una de las objetivos esenciales de la educación y la formación. Cabe destacar además que uno de los factores que subraya esta futura conjunción continental europea es precisamente la variable de la multiculturalidad.

Queremos destacar del proyecto de Educación de la UE ciertos aspectos meritorios como es su atención a la calidad, a la situación multicultural en desventaja, al fomento del entendimiento multicultural a través de las lenguas y al desarrollo real de los proyectos internacionales como es por ejemplo, el programa Erasmus.

Además la ponencia analiza el alcance de este proyecto entorno a la multiculturalidad como en efecto se extiende hasta los sectores de la cultura, la educación, la formación y 
el ocio participan del proyecto. Esto ha cambiado radicalmente las condiciones de acceso al conocimiento 0 a la adquisición del mismo, y en consecuencia contribuye al crecimiento económico, al desarrollo empresarial y al del empleo y potencia el desarrollo profesional, social y cultural, así como la creatividad y la capacidad de potencial económico y social del desarrollo de los contenidos digitales.

El escrito estudio la conveniencia y la problemática en su aplicación práctica social-con todos sus pros y sus contras- en el marco español educativo del que, por cierto, se tiene una mayor praxis desde que se puso en marcha estos proyectos y una nueva mentalidad entorno al fenómeno educativo y social que provoca la convivencia multicultural.

\section{PALABRAS CLAVE}

Educación - Multicultural - Intercultural - Sociedad - Unión Europea - mentalidad.

\section{ABSTRACT}

It is within the EU primal desire that any improvement in Education and training contributes to strengthening growth and competitiveness of Europe as Latinamerica notes U.S. and Europe. In the old continent is highlighted repeatedly the need to go beyond the purely economic aspects of development, but to consider the individual's personal fulfillment as one of the key objectives of education and training. It is also worth noting that one of the factors underlining this future European continental conjunction is precisely the variable of multiculturalism.

We want to highlight the proposed EU educations aspects as deserving their attention to quality, multicultural disadvantaged status, the promotion of intercultural understanding through the language and the actual development of international projects such as for example, Erasmus.

In addition, the paper discusses the scope of this project to the multicultural environment 
and in effect extends to the fields of culture, education, training and leisure sectors of the project. This has radically changed the conditions of access to knowledge or acquisition of it, and therefore contributes to economic growth, business development and employment, and enhancing the professional, social and cultural as well as the creativity and potential economic and social development of digital content.

The brief survey of convenience and practical problems in social, with all its pros and cons, the Spanish educational context which, incidentally, have more practice since he started these projects and a new mindset around the educational and social phenomenon that causes the multicultural coexistence.

\section{KEY WORDS}

Education - Multicultural - Intercultural - Society - European Union - Mentality

\section{ÍNDICE}

1. Introducción

2. Operatividad del proyecto en el contexto europeo

3. Dilemas entorno a la Multiculturalidad

4. Conclusiones

5. Bibliografía

6. Webgrafía

\section{Introducción}

Si atendemos a la génesis de la praxis intercultural europea, ya en su momento, 1973, el sociólogo Emile Durkeim había sentenciado la importancia de generar proyectos educativos en relación a la transformación social previsible y paralela: 
"Las transformaciones profundas a las que han sido sometidas o a las que se ven actualmente sometidas o alas que se ven actualmente sometidas las sociedades contemporáneas, requieren las transformaciones correspondientes dentro del campo de la educación nacional" (Durkheim,1973, 8)

El 29 de mayo de 1997 la Comisión adoptó una Comunicación relativa a la evaluación de la aplicación del Libro Blanco "Enseñar y aprender: hacia la sociedad cognitiva". Esta comunicación resume los principales mensajes políticos extraídos de los debates mantenidos con posterioridad a la publicación del Libro blanco.

Las formulaciones de la UE en principio las mueve la condición económica, pero posteriormente se descubre cómo se tiene en cuenta el desarrollo de cada ciudadano y el beneficio social (o bien común). La Comunicación sienta las bases de lo que podríamos llamar la Unión del conocimiento: se considera que cualquier mejora de la educación y la formación contribuye a reforzar el crecimiento y la competitividad de Europa; se destaca en repetidas ocasiones la necesidad de no limitarse a los aspectos puramente económicos del desarrollo, sino a considerar la realización personal del individuo como una de las finalidades esenciales de la educación y la formación.

Son numerosas las opiniones en favor de un modelo social alejado de la perspectiva de una sociedad escindida entre los que saben y los que no saben; numerosas tomas de posición apoyan una lucha reforzada en favor de la igualdad de oportunidades y hacen hincapié en las dificultades específicas de los grupos desfavorecidos.

Se reconoce ampliamente la necesidad de mejorar la convergencia entre enseñanza general y profesional, así como de valorar la formación profesional en su conjunto. Asimismo la construcción de la sociedad de la información (dominio individual de las técnicas de la comunicación) goza de aceptación general. Se reconoce de forma 
prácticamente unánime la competencia de los Estados miembros en materia de contenido y organización del sistema educativo y de la formación profesional en el respeto del principio de subsidiariedad y dejando abiertos nuevos espacios de cooperación a nivel comunitario. Son varias las opiniones que recuerdan el impacto decisivo de la pedagogía (mediación entre el individuo y el conocimiento), el papel de la familia y del entorno inmediato, así como el carácter primordial de la formación de docentes y formadores. Por su parte, el Parlamento se muestra particularmente preocupado por la disminución en términos relativos del gasto público en educación y formación.

Para combatir la exclusión, el Libro Blanco desarrolla, a título de proyecto experimental, el concepto de escuela de la segunda oportunidad. Ya se han puesto en marcha proyectos piloto en los Estados miembros, a los que seguirán otros hasta finales de 1997. El concepto debe responder a la gran diversidad de las estructuras sociales, económicas, culturales e institucionales de los países miembros.

Podemos destacar del proyecto de Educación de la UE su atención a la calidad, a la situación multicultural en desventaja, al fomento del entendimiento multicultural a través de las lenguas y al desarrollo real de los proyectos internacionales como Erasmus, calidad del sistema educativo, evaluación cualitativa de la educación escolar, acceso a la educación, educación de los hijos de emigrantes, escolarización de niños gitanos e itinerantes, o de padres que ejercen profesiones itinerantes, fomento de la enseñanza de lenguas y enseñanza de lenguas y proclamación del Año Europeo de las Lenguas 2001. Los programas que se han desplegado desde Sócrates en la primera etapa han sido Comenius, Erasmus y Lingua; en la segunda etapa se han añadido Grundtvig y Minerva. El programa Tempus se prevee en tres etapas. 


\section{Operatividad del proyecto en el contexto europeo}

Sin embargo hacemos notar la necesidad integradora de todo este proyecto educativo. Las disposiciones de aplicación de la Comisión son las siguientes. El 10 de enero de 2000 la Comisión ${ }^{1}$ presentó un informe sobre la aplicación del Libro Blanco «Enseñar y aprender - Hacia la sociedad del conocimiento». El objetivo de este informe es hacer un balance de la aplicación de los cinco objetivos que se ha marcado el Libro Blanco. Esto supuso la definición de tres grandes ejes en la construcción de la Europa del conocimiento: primero favorecer la contratación laboral de los jóvenes y de los trabajadores; después aumentar el potencial de los conocimientos; y por último, contribuir a la construcción de una Europa de los ciudadanos.

El informe revela que "en los cinco objetivos de la Comisión, se pudieron llevar a cabo experimentos que en algunos casos pueden dar lugar a un nuevo programa de acción comunitaria". Es el caso del Servicio Voluntario Europeo, que ilustra la solidaridad de los jóvenes con las personas desfavorecidas y les permite descubrir un itinerario propio de integración social.

Las Escuelas de la segunda oportunidad son otro aspecto positivo. Su objetivo es ayudar a los jóvenes en situación de exclusión a reintegrarse en la sociedad. En junio de 1999, bajo el impulso de la Comisión, se creó una asociación europea de las ciudades de Escuelas de la segunda oportunidad. Estas escuelas se han integrado en el Marco de acción de la Comisión Europea para un desarrollo urbano duradero en la Unión Europea.

En marzo de 1999 la fase experimental del proyecto de distintivo europeo para iniciativas que fomenten el aprendizaje de idiomas concluyó de forma satisfactoria en

\footnotetext{
${ }^{1}$ www.europarl.es (2002)
} 
Bruselas con un acontecimiento europeo en el que se presentaron 29 proyectos representativos del distintivo europeo. Como todos los participantes calificaron la experiencia de muy positiva, se prevé proseguirla en el año 2000 e incluso a más largo plazo.

Se expone la decisión del Consejo, de 22 de diciembre de 2000, por la que se adopta un programa plurianual comunitario de estímulo al desarrollo, el uso de contenidos digitales europeos en las redes mundiales y de fomento de la diversidad lingüística en la sociedad de la información. Otras medidas de este escrito parecen interesantes: Centros de lenguas, establecimiento de una red de centros de datos regionales, nacionales y por temas o disciplinas específicos, nuevos recursos, creación y distribución transnacional de nuevos conjuntos de datos para aquellas lenguas y ámbitos temáticos donde no existe ningún recurso conveniente.

Los sectores de la cultura, la educación, la formación y el ocio participan del proyecto. Esto cambia radicalmente las condiciones de acceso al conocimiento o a la adquisición del mismo, y en consecuencia contribuye al crecimiento económico, al desarrollo empresarial y al del empleo y potencia el desarrollo profesional, social y cultural, así como la creatividad y la capacidad de potencial económico y social del desarrollo de los contenidos digitales.

Se propone fomentar la diversidad cultural y el multilingüismo, particularmente en las lenguas de la Unión Europea, en los contenidos digitales de las redes mundiales, así como aumentar las posibilidades de exportación de las empresas europeas de contenidos, y en especial de las PYME, por medio de la adaptación cultural y lingüística a los destinatarios. Opta, por tanto, por garantizar la diversidad cultural, valorizar el patrimonio europeo y favorecer el acceso al conocimiento.

La consolidación de las infraestructuras lingüísticas de Europa entraña la institución de un marco abierto que incluya recursos multilingües e ínter funcionales tales como 
glosarios y antologías multilingües, memorias de traducción y recopilaciones terminológicas, así como las herramientas correspondientes como la traducción asistida por ordenador.

Ahora bien, si observamos la aplicación práctica en Europa es interesante la recomendación de una educación especial que aportan García Castaño, Moyano Pulido y Montes del Castillo. Se plantean la discusión sobre educación multicultural en el momento en que ciertos aspectos de la variable cultura, en tanto que se trata de una variable representativa de la diversidad, se introducen en el aula y en la escuela.

"Cuando existe una presencia de grupos étnicos claramente diferenciados por razones del color de piel, lengua materna, valores $y$ comportamientos religiosos, $y$, junto a todo ello y otros elementos más, diferencias socioeconómicas, se reconoce la necesidad de una educación "especial» para atender tales diferencias" (García Castaño y otros, 1997).

Son numerosas las direcciones en Internet en las que aparecen congresos de profesores, portales sobre el tema educativo o con sugerencias de proyectos o con experiencias al respecto. También hemos descubierto un crecido interés en el diseño de proyectos concretos para aplicarlos en el aula.

Según Azurmendi:

"Se diseñan programas que tratan de mejorar la situación de estos colectivos en las escuelas y que, en algunos casos, promuevan un respeto hacia su cultura de origen y una integración en la cultura de "acogida» (o al menos eso es lo que idealmente se pretende)". 
La educación intercultural en la escuela ha de preparar para vivir con los demás, con sus semejanzas y sus diferencias. La convicción de que la diversidad humana inherente a la unidad de la especie- ha de enriquecer la convivencia, no empobrecerla, debe guiar el proyecto educativo intercultural. La convivencia, no la mera coexistencia, nace de la aproximación cognitiva y afectiva a la realidad del otro y se manifiesta en la conducta social. En su polo positivo, estas tres dimensiones (cognitiva, afectiva y social) interconectadas son claves para impulsar y consolidar actitudes de respeto y colaboración entre culturas ${ }^{2}$.

Es interesante alguna postura en el ámbito educativo como la de Miguel Ángel Essomba quien destaca la perspectiva comunitaria que sale en ayuda de los centros educativos en dos vertientes: gracias a las "comunidades de aprendizaje" y gracias a las "ciudades educadoras" (Essomba, 2006, 95-97).

\section{Dilemas entorno a la Multiculturalidad}

El término intercultural se usa más en Europa y multiculturalismo más en América del Norte y parece ser de reciente aparición. Según Mikel Azurmendi «Multiculturalismo» es un concepto relativamente nuevo que no expresa que existan muchas culturas en el mundo, ni tampoco que existan muchas en convivencia en un sólo país, sino que

"fue pensado para referir un Estado-nación democrático cuyo pluralismo debía consistir en promover diferencias étnicas y culturales. Seguramente quien primero lo acuñó fue el Gobierno canadiense para referirse a su nueva política de finales de los años $60^{\prime 3}$.

\footnotetext{
${ }^{2}$ Mikel Azurmendi, 2002

${ }^{3}$ I dem
} 
Dos autores emblemáticos sobre el multiculturalismo Ch. Taylor y W. Kymlicka han sido criticados pues conciben la cultura como un cuerpo uniforme, con fronteras delimitadas hacia el exterior y con homogeneidad interna en cuanto a valores ${ }^{4}$.

La clave de la conveniencia de la educación en multiculturalidad quizás también radique en lo necesidad de retroalimentación que mantiene la sociedad actual tal y como señala Durkeim:

"Muy lejos de que la educación tenga por objetivo único o principal al individuo y sus intereses, ante todo es el medio a través del cual la sociedad renueva de continuo los condicionamientos de su propia existencia. La sociedad no puede vivir más que si existe entre sus miembros una homogeneidad suficiente" (Durheim,1974, 106).

La multiculturalidad tiene su doble cara, digamos que por una parte enriquece nuestra visión de la realidad y de la verdad, y nos posibilita la comunicación con otras personas ${ }^{5}$, pero por otra parte si no estamos intelectualmente preparados, puede debilitar nuestra propia identidad cultural, y si apreciamos nuestra identidad religiosa a esta todavía más.

Según Esperanza Sáez Pineda:

"La educación multicultural pretende hacer del alumno un sujeto respetuoso e interesado en la diversidad humana. Este tipo de enseñanza intercultural tiene como objetivo el conocimiento sobre "el otro" o sobre la inmigración y las minorías que conviven con él diariamente. Pero no ha de servir sólo para poner de manifiesto lo diferentes que somos, para remarcar las diferencias entre grupos

\footnotetext{
${ }^{4}$ Raquel Kritsch, 2009

${ }^{5}$ Esperanza Sáez Pineda, 2002
} 
étnicos, sino para modificar y crear nuevas actitudes integradoras, para profundizar en los valores de cada uno y respetarlos."

Hemos encontrado todo tipo de opiniones favorables y críticas sobre su aplicación en la UE. Una opinión desfavorable es la de Jagdish S. Gundara ${ }^{6}$.

A veces el diagnóstico es un poco pesimista sobre la sociedad actual. Según García Castaño y otros:

"En las sociedades actuales se observa una tendencia a hacer absolutas las culturas locales, regionales y nacionales con objetivos políticos, nacionalistas e independentistas, o simplemente con finalidades de reforzamiento de la propia identidad, en un marco de luchas políticas y de redistribución del poder" (García Castaño y otros, 1997).

Piensan que al mismo tiempo, se aprecia una tendencia contraria que se manifiesta en procesos de homogeneización y estandarización de la cultura bajo la influencia, las directrices y los intereses de las multinacionales de la información y de la comunicación y de sus industrias culturales transnacionales.

Frente al auge de los nacionalismos, de países que reivindican su identidad y de la importancia de sentirse identificado con una etnia o grupo en concreto, Esperanza Saéz opina que aumentan paradójicamente las sociedades cada vez más pluriétnicas, más pluriculturales. Esta situación provoca en más una ocasión tensiones entre los habitantes de un país. El fracaso a la hora de abordar estos conflictos es lo que provoca en muchas ocasiones situaciones como las que se están viviendo entre israelíes y palestinos, o la antigua Yugoslavia. Piensa que el multiculturalismo es una realidad bien visible en la sociedad actual, es un fenómeno unido al de la

\footnotetext{
6 Jagdish S. Gundara, 1997
} 
inmigración, y viene dado a través de la convivencia entre personas de diferentes culturas dentro de un mismo espacio. Por eso cree que la educación intercultural es la clave para conseguir que realmente se respete el derecho de toda persona a la dignidad y a la libertad de creencias, sin distinción de sexo, cultura, religión o lengua ${ }^{7}$.

A su vez unido al fenómeno de la multiculturalidad se une el fenómeno de la globalización que lo potencia, facilitado por los Media. Es interesante lo que aporta Alejandro Llano sobre las ventajas: facilita la comunicación y la conciencia social: la mundialización como fenómeno nos une de forma impensable tan sólo hace una década, nos aproxima por la facilidad de los medios de transporte y las nuevas tecnologías de la comunicación. Piensa que contribuye al humanismo cívico el hecho de conocer mejor las necesidades de pueblos con carestías, y la creación de canales de ayuda, así como la proliferación de foros que atienden estos problemas ${ }^{8}$.

El filósofo opina que es ventajosa la globalización si la orientación de ésta se dispone al servicio del conocimiento, y no tanto de la información por sí misma porque supone una elevación del nivel educativo y cultural, un crecimiento en el ser de las personas ${ }^{9}$. Se trata de una fuente y recurso de poder que según Naisbitt no sólo es renovable sino generable. Su fomento potencia la ulterior producción de riqueza y pone metas más altas al hombre, pues, como opina Alejandro Llano:

"Las virtudes cognoscitivas y prácticas representan el único modo de no perder la propia vida, de que el tiempo vital no se escurra como

\footnotetext{
${ }^{7}$ Esperanza Sáez Pineda, 2000

${ }^{8}$ Alejandro Llano, 2002, 57 y 98

${ }^{9}$ Idem, 100
} 
agua entre las manos, sino que se remanse en la forma de ser más, y, en consecuencia, de ser capaz de más" (Llano, 2002, 62-63).

También expone las desventajas que valora del fenómeno de globalización como es el predominio monocéntrico de Estados Unidos y países satélites donde no hay retroalimentación, ni participación en el poder por parte de otros. Visto así puede tomarse como un medio en el que los poderosos se pueden aprovechar de los débiles, pero también pueden llevarse la sorpresa de que se despierte la creatividad en aquellos que antes no tenían la posibilidad de expresarlo. Además encuentra que el fenómeno en realidad no alcanza a tanta población por los estudios que se han realizado, pues sólo alcanza a un $15 \%{ }^{10}$.

Otro factor que expone como consecuencia de la globalización es el peligro del relativismo cultural y moral. Ante la diversas culturas, Llano propone como referencia ética a la persona humana:

"El relativismo cultural y moral comparece cuando se estudia el comportamiento humano a partir de los diversos estilos de vida $y$ criterios de valoración aplicados por las diferentes culturas, las cuales resultan irreconciliables si no se reconducen a allí de donde toda cuestión ética surge y a donde toda cuestión ética retorna, es decir, será por tanto en la propia persona humana" (Llano, 2002, 199).

Además explica la diferencia entre el pluralismo democrático y relativismo cultural, para sugerir la conveniencia del primero y la inconveniencia y error del segundo planteamiento. Si en la democracia se da el pluralismo porque se reconoce los diversos caminos que la libertad sigue en su búsqueda de la verdad práctica, cuando acontecen posiciones diversas que entran en diálogo, e incluso en conflicto, "es

\footnotetext{
${ }^{10}$ Idem, 99-100
} 
precisamente porque se parte del convencimiento de que hay realmente verdad y de que no es vana ni utópica la esperanza de acceder a ella por el correcto ejercicio de la inteligencia práctica."(Llano, 2002, 152). En cambio el relativismo cultural juzga las acciones de forma subjetiva, por el modo de usarlas según esa cultura, y ya no hay un bien apresable a través de la razón en el que nos podamos poner de acuerdo ${ }^{11}$.

La postura que se considera en este estudio, como más razonable, es el respeto sin duda de otra forma de pensar en cuanto a lo religioso, pero haciendo defensa de lo propio, con actitud de diálogo y apertura mental de esa verdad que está en el otro planteamiento; y además con una actitud de búsqueda de la verdad tanto de la propia tanto de la ajena excluyendo el miedo a conocer lo ajeno, pues, sería indicio de que no se está del todo convencido de que nuestro punto de vista sea más acertado, o supondría en todo caso otro indicio de que no hemos buscado argumentos que hagan razonable nuestro planteamiento; es decir, quizás ni hemos sido críticos, ni hemos profundizado, ni hemos visto que en la práctica nos lleve a un bien excelente, o ni siquiera eso, nos debemos a la comodidad de quedarnos en nuestro mundo.

Se puede pensar que hay parte de verdad (según una gradación y acercamiento a la verdad de las cosas según su naturaleza y a lo que Dios haya establecido) en cada planteamiento religioso. Es legítima la razonabilidad de su defensa, la conservación y la transmisión por parte de cada uno, así como defensa de la libertad de conciencia ante todo. Igualmente cualquier manifestación cultural puede enriquecer más según corresponda a esa medida de lo excelso de la naturaleza del Ser, y manifieste el gran potencial del hombre. Por tanto el factor religioso es necesario y está vinculado a una mayor riqueza cultural ganada por el hombre a lo largo de los siglos:

\footnotetext{
${ }^{11}$ Idem, 153
} 
"Se puede contemplar (...) todo cuanto es bello, bueno y verdadero. Pero todo esto quedaría sin finalidad y sin fundamento si no le fuera posible al hombre abismarse en la contemplación de Dios. Esto es algo que, de un modo $u$ otro han sabido todas las culturas $y$ religiones" (Llano, 2002, 163).

La educación multicultural según García Castaño y otros:

"debe ser la potenciación, desde la escuela y otras instancias educativas, de una reflexión social (de la que emergen varios discursos), de la autocomprensión de los grupos humanos y la autocrítica de las propias formas culturales, (...) con el objeto de mejorar sus propias condiciones de vida y afianzar su propia identidad cultural bajo el reconocimiento y la aceptación de la diversidad cultural" (García Castaño y otros, 1997).

Cara a una aplicación práctica, hay autores que acuden al recurso de la tolerancia. Estamos de acuerdo con la definición de Alfonso Aguiló sobre la tolerancia: "respeto y consideración hacia la diferencia, como una disposición a admitir en los demás una manera de ser y obrar distinta a la propia, o como una actitud de aceptación del legítimo pluralismo, es a todas luces un valor de enorme importancia" (Aguiló, 1995, 9).

Este estudioso piensa que propagar en este sentido la tolerancia puede contribuir a resolver muchos conflictos y a erradicar muchas violencias ciudadanas, por tanto, se hace necesario y urgente. Sin embargo, promover una acertada aplicación de la tolerancia le parece que es algo extremadamente difícil y complejo. Recuerda que la tolerancia ha de tener unos límites. Como ha señalado Norberto Bobbio, la verdadera tolerancia se fundamenta en la firmeza de principios, que se oponen a la indebida exclusión de lo diferente. Apunta que el problema surge cuando esa diversidad deja 
de ser legítima, o entra en colisión con el bien común, o con los derechos de los demás, y entonces se trata de una tolerancia del mal, que sufre alguna falta de bien. El terreno que hay determinar entonces es el límite de lo no tolerable: en principio la legítima diversidad siempre debe tolerarse, pero la ilegítima puede tolerarse 0 no según los casos porque se pone en juego algún bien básico o bien común. Por ejemplo, dice que a nadie se le ocurre tolerar acciones como el robo, la violación o el asesinato. Ignacio Sánchez Cámara pone otros ejemplos que atentan contra la dignidad humana como la permisión de los sacrificios humanos, o los malos tratos ${ }^{12}$. Alfonso Aguiló critica la postura de defensa de valores relativos, pues considera que es una postura contradictoria de por sí. Sí admite distintos valores en las distintas culturas, pero defiende que unos responden más a la dignidad humana que otros. ${ }^{13}$

Otros autores se preocupan de su aplicación, por ejemplo, la que previene de que se parta de un sentido negativo de la multiculturalidad ${ }^{14}$. En este sentido Margarita Bartolomé comenta:

"Los centros educativos deben centrarse en el reconocimiento multicultural, no en la tolerancia. La tolerancia es asimétrica, ser capaz de aceptar, convivir con personas que no son como yo. La multiculturalidad no se abordar desde una perspectiva negativa."

Otra postura ante el tema es la de J. Martínez de Pisón. Postula que la tolerancia no es suficiente, y en cambio propone el acceso a la multiculturalidad desde los recursos que busque el Estado de Derecho:

\footnotetext{
12 Ignacio Sánchez Cámara, 2002

13 Alfonso Aguiló, 1995, 9-32

${ }^{14}$ Margarita Bartolomé, 2000
} 
"Hoy por hoy el Estado de Derecho debe utilizar otros instrumentos más acordes con su evolución para resolver las tensiones $y$ problemas que el "choque de civilizaciones", (...) es específico de las sociedades de finales del siglo XX y principios del XIX" (Martínez de Pisón, 2001, 23-24).

Por su parte Esperanza Pineda propone como principio básico el respeto:

"Aceptar el multiculturalismo no supone tener que compartir las creencias de una determinada sociedad para sentirse integrado en una cultura. Para sentirse integrado en una cultura uno se tiene que sentir respetado y para ello basta que el grupo que comparte un espacio cumpla las normas del estado de derecho, sin necesidad de compartir la misma cultura" (Pineda, 2000).

La piedra de toque de la multiculturalidad es el día a día de la convivencia, o sea, la adaptación de las personas entre sí, donde se pone en juego la capacidad relacional de la persona. Valentín Otero comenta que la convivencia multicultural es una ocasión para favorecer el enriquecimiento educativo y la unidad humana:

"Resultaría de todo punto empobrecedor, cuando no claramente perverso, limitar la capacidad de apertura del educando a ciertos grupos culturales. (...) El proceso educativo, hoy más que nunca, debe fortalecer su compromiso con el "ecumenismo" o unidad humana" (Martínez-Otero, 2002 ) $^{15}$.

\footnotetext{
${ }^{15}$ Es doctor en Psicología y en Pedagogía, y profesor de la Universidad de Madrid y del Centro Universitario "Don Bosco".
} 
En un artículo del periódico narraban el testimonio de dos niños (uno cristiano y otro musulmán) que sabían conjugar el multiculturalismo a través de la amistad y de formar parte en el mismo equipo de fútbol del barrio ${ }^{16}$.

Se trata de un reto educativo, pues, son muchos los caminos y grados para entenderse, por ejemplo, la capacidad empática que se favorece resaltando la educación en valores entorno a la convivencia social. Afortunadamente hemos encontrado muchas referencias prácticas de cómo saber convivir en la escuela por parte del profesorado y cómo se promociona la multiculturalidad como valor entre los alumnos. García Castaño y otros explican que beneficia a los alumnos en cuanto aprender a perder estereotipos: "En el desarrollo de la educación multicultural, ello se traduce en una contribución a la eliminación de la tendencia a estereotipar a los estudiantes de acuerdo con sus identidades" (García Castaño, 1997).

Este estudio considera que también puede ser beneficioso la adquisición de una nueva sensibilidad, la conciencia de una mayor capacidad de comunicación, y la apertura mental cultural, así como que se favorezca el valor de la solidaridad con otros pueblos y culturas. Alejandro Llano piensa que la sociedad se halla ante un momento de sensibilización sobre el valor de la solidaridad:

"Está emergiendo desde hace algunos años una nueva sensibilidad que reclama bienes comunicativos, no disgregadores sino solidarios. El campo de juego interpersonal se vitaliza y se expande, de modo opuesto al estrechamiento que acontece en los bienes excluyentes" (Llano, 2002, 62).

Es interesante lo que plantean García Castaño y otros sobre el concepto de cultura y la deficiencia que guarda con respecto a las minorías porque arroja reflexiones sobre

${ }^{16}$ La Vanguardia, 22.VII.2002 
la propia complejidad de lo multicultural que hemos percibido a lo largo de la elaboración de este escrito ${ }^{17}$. Hacen una revisión de los planteamientos que se hacen en educación. Les parece que en la mayoría de las visiones sobre educación multicultural se halla implícita una concepción algo estática de la «cultura». La cultura es vista como un conjunto más o menos implícito de características inmutables atribuibles a grupos diferentes de personas. Éstas son usadas para identificar a la gente y, a menudo, para producir estereotipos, en contra de la intención (Rosen, 1977). Critican que la concepción de cultura expuesta por el Libro IV de la Royal Comisión $(1969,11)$ como idea final, bajo el epígrafe «Las contribuciones culturales de otros grupos étnicos», revela una ficción que se asemeja poco a la realidad de las minorías. Exponen que la concepción de «La Cultura», glosaba la Comisión, "es una forma de ser, pensar y sentir. Es una fuerza rectora que anima a un grupo significativo de individuos unidos por una lengua común que comparten las mismas costumbres, hábitos y experiencias".

Estos autores hacen notar que el multiculturalismo de por sí presenta una fragmentación incluso interna, otro dato más para ponderar que se trata de un fenómeno poliédrico, pues, si el estudio se dispone desde el punto de vista subjetivo, es decir, conformado según la propia percepción del individuo se fragmenta: "Cada miembro tiene una versión personal de cómo funcionan las cosas en un determinado grupo y, de este modo, de su cultura. Lo que se presenta ante nosotros como la cultura de ese grupo no es otra cosa que una organización de la diversidad, de la heterogeneidad intragrupal inherente a toda sociedad humana." Lo que cada uno percibe será una versión de su "propiospecto": "Su versión personal de la cultura 0 , con el término que acuñó Goodenough, su propiospecto, es la totalidad de esas «parcialidades» que conforman una visión privada, subjetiva del mundo y sus

\footnotetext{
${ }^{17}$ García Castaño y otros, 1997
} 
contenidos, desarrollada a lo largo de su historia experiencial" (García Castaño y otros, 1997).

\section{Conclusiones}

Esta disertación piensa que el multiculturalismo consiste en un fenómeno complejo al que la sociedad y la educación no ha hecho más que empezar a enfrentarse, y sobre el que tendrá que desplegar muchos medios educativos y estructuras prosociales durante décadas, pues, se trata de un proyecto ambicioso de transformación de la mentalidad cultural europea.

\section{Bibliografía}

AGUILÓ, Alfonso. (1995). La tolerancia. Palabra. Madrid.

AZURMENDI, Mikel. "La invención del multiculturalismo", ABC digital, 18.III.2002

BARTOLOMÉ PINA, Margarita. "De la educación multicultural a la construcción de la ciudadanía" en Seminario: "La atención a la diversidad. La escuela intercultural", Madrid, Universidad a Distancia, UNED

http://www.mec.es/cesces/seminario1999-2000.htm [acc: 3.09.2002]

GARCÍA CASTAÑO, Francisco J.; PULIDO MOYANO Rafael A. y MONTES DEL CASTILLO, Ángel. Biblioteca Digital de la OEl, "Multiculturalismo. "La educación multicultural y el concepto de cultura" en Revista Iberoamericana de Educación, no. 13, enero-abril 1997, Educación Bilingüe Intercultural, (Organización de Estados Iberoamericanos para la Educación de la Ciencia y de la Cultura) www.oei campusoei.org/oeivirt/multi.htm - 5k [acc: 1.09.2002]

DURKEIM, Emile. (1996). Educación y sociología. Península. Barcelona. 
Educación Intercultural

(Página web de la minoría gitana)

www.pangea.org/aecgit/educacion.htm - 29k [acc: 1.09.2002]

ESSOMBA, M. A. (2006). Liderar escuelas interculturales e inclusivas. Equipos directivos y profesorado ante la diversidad cultural y la inmigración. Grao. Barcelona.

Interculturalismo y educación

Joaquina CABELLO HIDALGO, Proyecto de investigación sobre el polígono de la Cartuja, Granada, en Gazeta de Antropología, n.10, 1993

www.ugr.es/ pwlac/G10_10] oaquina_Cabello_Hidalgo.html [acc: 1.09.2002]

KRITSCH, R. (2010). Kant, Burke y los derechos del hombre en Pedro Aullón de Haro, Teoría del Humanismo. Verbum. Madrid.

LLANO, Alejandro (2002). La vida lograda. Ariel. Barcelona.

MARTÍNEZ DE PISÓN, J. (2001). Tolerancia y derechos fundamentales en las sociedades multiculturales. Tecnos. Madrid.

MARTÍNEZ-OTERO PÉREZ, Valentín. "La mirada intercultural en la escuela", 13. III. 2002, en Comunidad Escolar, Periódico digital de información educativa, Año XX, n. 695

http://comunidad-escolar.pntic.mec.es/695/tribuna.html [acc: 2.09.2002]

Multiculturalismo, minorías I slam en Europa

Discurso pronunciado por Giovanni MANGION en el II Congreso Europeo del Consejo islámico para la Cooperación en Europa, celebrado en Toledo, España, 29-30 Octubre 1998

www.islamhispania.com/_opinion/multiculturalismo.htm - [acc: 1.09.2002]

Portal institucional de la UE

www.europarl.es [acc: 1.09.2002] 
Revista española de Pedagogía, n. 201, mayo-agosto 1995, (Año internacional de la tolerancia)

www.ucm.es/info/quiron/repinpro.htm - 76kwww. [acc: 1.09.2002]

RUEDA BARRERA, Eduardo Alfonso. "Polifonía y concierto del Nosotros". Multiculturalismo e identidad nacional diciembre 1998

www.colciencias.gov.co/seiaal/ congreso/Ponen9/RUEDA.htm [acc: 1.09.2002]

SÁEZ PINEDA, Esperanza. "Educación Intercultural ". Instituto Superior de Estudios Psicológicos ISEP, 15 de noviembre del 2000, n. 3 www.educaweb.com/esp/servicios/monografico/multiculturalidad/article2.asp [acc: 1.09.2002]

SÁNCHEZ CÁMARA, Ignacio. "Multiculturalismo contra integración", ABC digital, 5.11 .2002

SANTOS REGO, M. (1994). "Teoría y práctica de la educación intercultural. ... Pluralismo, multiculturalismo y extranjeros", tesis doctoral, Madrid, Taurus. www.mec.es/sgpe/docs/margarita_bartolome_bliliografia.pdf $\quad$ - Similar pageswww.fund-culturadepaz.org/spa/04/cent0402f.htm [acc: 3.09.2002]

S. GUNDARA, Jagdish. "Diversidad social, educación e integración europea", Centre for Multicultural Education, Institute of Education, University of London, Revista Iberoamericana de Educación n. 13, enero-abril 1997

http://www.oei.org.co/oeivirt/rie13.htm [acc: 1.09.2002]

Virtual forum Intercultural Communication

Comunicación intercultural, Multiculturalismo y desigualdad social, John DAWNING y Charles HUSBAND (Portal Canadiense), www.portalcomunicacion.com/forumv/ forum1/text/esp/text2 [acc: 1.09.2002] 